\title{
«Wir wissen nie, was kommt»
}

\author{
Wenn zwei Kinder schwer krank sind, stellt dies das Leben einer Familie vollständig \\ auf den Kopf. Für die Mutter bedeutet das, dass sie fast rund um die Uhr gefordert \\ ist. Eltern wie Kinder leben mit der Angst, dass stets etwas Unvorhergesehenes \\ passieren kann.
}

Mütter hören alles, wenn es um ihre Kinder geht. Sie wachen auf, wenn die Kleinen mitten in der Nacht schreien. Und sie verstehen ihre Kinder auch dann, wenn diese Mühe mit dem Sprechen haben. Silvan ist doppelt gehandicapt. Der Achtjährige muss gegen die Geräusche eines Atemgeräts ankämpfen, ein regelmässiges Blasen und Saugen. Zusätzlich eingeschränkt ist er durch die Atemmaske, die in einen Helm integriert ist und das meiste von dem verschluckt, was Silvan sagt.

«Mama, ich habe heute schon fünf Kordeln gemacht», sagt er stolz. Seine Mutter strahlt und lobt ihn für die schönen Farben, die er ausgewählt hat. «Ich mach noch mal drei, auch eine für Nadja», kündigt Silvan an. Für den zarten Jungen bedeutet das Drehen von Kordeln aus Wollschnüren Schwerstarbeit. Silvan ist muskelkrank. Die Muskeln in Fingern und Armen sind so schwach, dass er nicht mehr selbständig essen kann. Laufen kann er seit zwei Jahren nicht mehr - seither benötigt er einen Rollstuhl.

\section{Plötzlich wollte er gar nicht mehr laufen und klagte über schmerzende Beine.}

Auch seine kleine Schwester Nadja kann nur noch laufen, wenn sie festgehalten wird. Die meiste Zeit sitzt die Fünfjährige ebenfalls im Rollstuhl. Das dritte Kind von Judith und Christian Weber, der zehnjährige Roman, ist gesund. «Er wünscht sich nichts mehr, als dass seine Geschwister auch gesund wären», sagt der Vater. Die Chancen auf Heilung stehen schlecht. Beide Kinder leiden unter einer bislang unbekannten Variante von Muskeldystrophie (siehe Kasten), bei der die Muskeln in den Beinen und Armen schwinden, während sich die Brustmuskulatur versteift, was die Atmung erschwert. Auch Nadja ist auf eine Atemmaske angewiesen; derzeit aber nur in

\section{«Nous ne savons jamais ce qui nous attend»}

Lorsque deux enfants sont gravement malades, c'est toute la vie de famille qui s'en trouve bouleversée. Chez la famille Weber, tout tourne autour de Silvan, huit ans, et Nadja, cinq ans, tous deux atteints de dystrophie musculaire, une maladie qui les empêche de marcher. Ils ont besoin d'aide pour s'habiller, pour manger, et ne peuvent pas aller tous seuls à l'école ou au jardin d'enfants comme n'importe quel enfant en bonne santé. Le troisième enfant de Judith et Christian Weber, Roman, dix ans, est lui en bonne santé. «ll ne souhaite rien de plus que de voir son frère et sa sœur en bonne santé comme lui», déclare le père. Mais les chances de guérison sont minimes. Parents et enfants vivent constamment dans la peur que quelque chose d'imprévu survienne. «Nous ne savons jamais ce qui nous attend», explique la mère. Silvan attrape sans arrêt de graves infections et a de plus en plus de peine à respirer. Nadja doit elle aussi porter un masque respiratoire pendant la nuit. Christian accepte mal l'impuissance de la médecine face à la maladie qui touche ses enfants: «Aujourd'hui, on peut aller sur la Lune mais personne ne peut aider mes enfants.»

der Nacht. Silvan benötigt auch am Tag immer wieder stundenweise Atemunterstützung.

«Es muss ja irgendwie weitergehen», sagt die Mutter und streicht sich Tränen von der Wange. Der Alltag ist für die ganze Familie äusserst beschwerlich. Die beiden kranken Kinder erfordern extrem viel Aufmerksamkeit. Silvan benötigt Betreuung fast rund um die Uhr. Am Morgen muss er gewaschen und bewegt und über eine Magensonde ernährt werden. Er braucht Hilfe beim Inhalieren und beim Abhusten 
von Schleim und muss schliesslich für die Schule parat gemacht werden. Nadja braucht ebenfalls Hilfe beim Waschen, Anziehen und Frühstücken - um 8.00 Uhr wird sie für den Kindergarten abgeholt. Wenn die Kinder um 12.00 Uhr nach Hause kommen, gibt es Mittagessen. Weil Silvan so gerne isst, füttert ihn die Mutter am Mittag und am Abend. «Eine Stunde brauchen wir mindestens dafür», sagt sie. Am Nachmittag steht für beide Kinder Physiotherapie auf dem Programm. Findet die Behandlung ausser Haus statt, müssen die Kinder jeweils hingebracht und wieder abgeholt werden.

\section{Das Ehepaar kämpft seit Jahren, seinen Bauernhof rollstuhlgerecht umbauen zu dürfen.}

Bis vor kurzem haben die Eltern, beide 40 Jahre alt und im eigenen landwirtschaftlichen Betrieb tätig, fast alle Betreuungsarbeiten alleine übernommen. Nun kümmert sich die Spitex während 30 Stunden pro Woche um Silvan, für Nadja müssen 1 11/2 Wochenstunden genügen. Die Invalidenversicherung bezahlt für beide Kinder Hilflosenentschädigung, für Silvan den höchsten, für Nadja den mittleren Satz sowie einen Intensivpflegezuschlag für Silvan. Zwei bis drei Mal pro Woche kommt für wenige Stunden eine Haushaltshilfe, um die dringendsten Arbeiten zu erledigen.

Weitere Unterstützung bekommen die Webers kaum - die Angehörigen wohnen entweder zu weit weg oder finden keine Zeit dafür. Im Gegenteil hört die Familie häufig Kommentare wie «die Spitex kümmert sich ja, Silvan ist doch mit seinem Rollstuhl ganz mobil...». Als wären das nicht genug Hindernisse, kämpft das Ehepaar seit Jahren mit den Behörden darum, seinen Bauernhof rollstuhlgerecht umbauen zu dürfen. «Das Schreiben von Briefen an Behörden kostet am meisten Energie», sagt Christian Weber. Auch hier fehlt es der Familie an Hilfe, die sie dringend benötigen würde.

\section{Muskeldystrophie}

Diese Gruppe von Muskelerkrankungen hat eine genetische Ursache. Meist werden sie vererbt, selten sind es spontane Mutationen. Diese führen dazu, dass die Muskelzellen allmählich beschädigt werden, was zur vollständigen Zerstörung der betroffenen Muskeln führen kann. Die Krankheitsbilder, welche die Skelettmuskulatur betreffen, sind sehr unterschiedlich. Muskeldystrophien sind äusserst seltene Erkrankungen, mit einer Häufigkeit von 10 Erkrankten auf 100000 Personen. Die häufigste Muskelerkrankung im Kindesalter ist Duchenne Muskeldystrophie, von der fast ausschliesslich Jungen betroffen sind. Eine ursächliche Behandlung gibt es bislang nicht; aktuell werden verschiedene Therapieansätze erforscht. Mit Physiotherapie, Schienenbehandlung, Operationen und Medikamenten gelingt es, die Symptome zu lindern und den Verlauf zu verzögern.

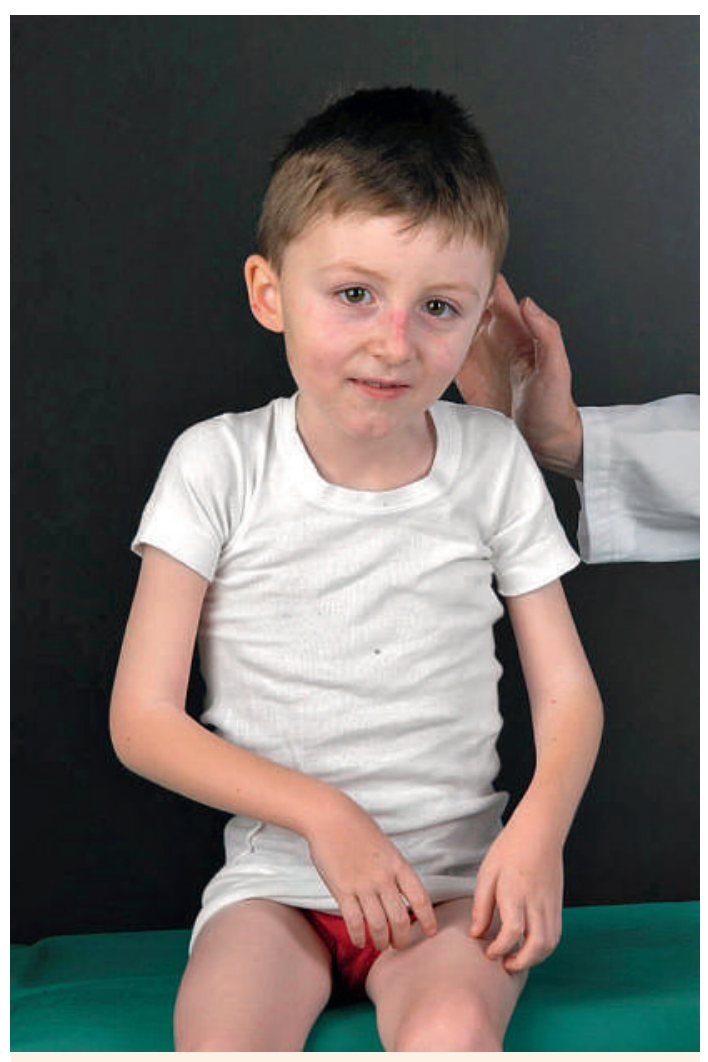

Aus kleinen Dingen Kraft schöpfen: Der achtjährige Silvan leidet an einer Muskeldystrophie und braucht durchgehend Betreuung.

Doch ihre grösste Sorge gilt den beiden kranken Kindern. Silvan war $2 \frac{1}{2}$ Jahre alt, als seine Schwester Nadja geboren wurde, und die Mutter merkte, dass etwas nicht stimmte. Dass er erst spät mit dem Laufen anfing, hat Judith Weber zunächst nicht beunruhigt: «Ich dachte, er braucht einfach ein bisschen länger.» Doch dann wollte er plötzlich gar nicht mehr laufen und klagte über schmerzende Beine. Eine Blutuntersuchung zeigte hohe Kreatinkinase-Werte, ein klares Indiz für eine Schädigung der Muskelzellen. Der Arzt sprach von Muskelentzündung und verabreichte Schmerzmittel. Eine Biopsie brachte schliesslich Gewissheit, dass Silvan an einer Muskelerkrankung litt. Als Nadja vier Monate alt war, wurde auch bei ihr Muskelschwund diagnostiziert. Für die Familie war das ein «Riesenschock», zumal Muskelerkrankungen bei Mädchen eher selten sind und ähnliche Erkrankungen weder aus der Familie der Mutter noch aus der des Vaters bekannt waren.

Nadja ist ein aufgewecktes, kleines Mädchen, das «gerne und viel redet», so der Vater, und sich jeden Tag auf den Kindergarten freut. Später soll sie eine Regelschule besuchen können, hoffen die Eltern. Silvan besucht eine Klasse im Zentrum für Körperbehinderte. Auch er ist gerne mit anderen Kindern zusammen, aber er hat zunehmend Angst, dass ihm dort nicht geholfen werden kann, wenn er in Atemnot gerät. Die Mutter quält sich jeden Morgen mit 
dem Entscheid, ob es ihm heute so gut geht, dass sie ihn gehen lassen kann. Immer wieder erreicht sie dann trotzdem ein Anruf, dass sie sofort kommen muss. Wenn Silvan nicht zu Hause ist, ist Judith Weber unruhig. «Ich bin nie ohne Handy unterwegs», sagt sie. Und stets muss sie darauf gefasst sein, von einem Moment auf den anderen alles stehen und liegen zu lassen. Angst treibt die ganze Familie um: «Wir wissen nie, was kommt», sagt die Mutter. riert den Hof mit wechselnden Ausstellungen von dem, was die Felder hergeben: Kürbisse im Herbst, Melonen im Sommer. Zeit für sich selbst findet sie kaum. Geht sie abends mal weg, kann sie's kaum geniessen, weil sie weiss, dass Silvan nervös ist, wenn sie nicht im Haus ist.

Die Nächte sind häufig unruhig; beide Kinder sind mit einer Klingel ausgestattet. Silvan betätigt seine jede Nacht, meist mehrmals. Dann steht die

\section{Die Mutter quält sich jeden Morgen mit dem Entscheid, ob es ihm heute so gut geht, dass sie ihn zur Schule schicken kann.}

Im letzten halben Jahr ist Silvan sehr häufig krank gewesen. Wegen verschiedener Infekte hat er Monate im Kinderspital Zürich verbracht, viele Wochen davon auf der Intensivstation. Nun hoffen die Eltern, dass er sich stabilisiert hat und regelmässig in die Schule kann. «Er lernt so gerne und bräuchte mehr Förderung», findet die Mutter. Neu hat er das Schachspielen entdeckt; sein Vater spielt mit ihm, so oft er kann. Mehr als für ihre Kinder da zu sein, können die Eltern kaum tun. Dass die Medizin machtlos, ist, kann der Vater nur schwer verstehen: «Man kann heute auf den Mond fliegen, aber meinen Kindern kann man nicht helfen.»

Die Familie hat sich in ihr Schicksal gefügt. «Wir sind schon zufrieden, wenn wir's daheim gut bewerkstelligen können», sagt Christian Weber. Grössere Ausflüge oder gar ein Urlaub sind kaum möglich. Einmal sind sie für drei Tage ins Tessin gefahren, das Auto vollgepackt mit Rollstühlen und Pflegeutensilien für die Kinder. «Das war ein Riesenstress», sagt die Mutter. Trotzdem hätten sie es genossen, zu fünft einen Ausflug machen zu können. Es sind kleine Ereignisse, aus denen die Familie Kraft schöpft. Im Sommer durften Nadja und Silvan beim Zirkus «Wunderplunder» mitmachen - Bilder zeigen vor Glück strahlende Kinder.

Christian Weber findet seine Ruhe, wenn er auf den Feldern unterwegs ist. Seine Frau muss ihre Oasen der Ruhe bewusst suchen. Morgens steht sie extra früh auf, um alleine frühstücken zu können. «Ich geniesse das», sagt sie. Gelegentlich geht sie mit raus aufs Feld, sie organisiert den Hofladen und deko-
Mutter auf, gibt ihm zu trinken oder hilft ihm beim Abhusten von Schleim. Vor allem tröstet sie ihn. Manchmal ist Judith Weber so müde, dass sie die Klingel nicht hört. Dann steht der Vater auf. Meist muss er seine Frau dann doch wecken. Sie ist die Einzige, die ihren Sohn beruhigen kann, wenn er nicht mehr schlafen kann.

\section{Zürcher Ärzteball am 12. Mai 2012 im Dolder Grand zugunsten des Neuromuskulären Zentrums Zürich}

Die Zürcher Ärztegesellschaft lädt alle Ärztinnen und Ärzte und deren Freunde zu einer exklusiven Ballnacht ins Dolder Grand ein. Der 2. Zürcher Ärzteball ist der glamouröse gesellschaftliche Höhepunkt des Jahres und findet am 12. Mai 2012 statt. Ein Feuerwerk mit der Dani Felber Band, «Helga is Bag» (ehemals Acapickels) und einer schwindelerregenden Mitternachtsshow erwartet Sie. Die Tombola hat Preise im Wert von über 60000 Franken. Der gesamte Erlös dieses Benefizballs kommt dem Neuromuskulären Zentrum Zürich zugute. Bereits vor zwei Jahren konnten über 220000 Franken überwiesen werden.

Verpassen Sie nicht, sich bald anzumelden. Die Plätze sind limitiert und werden nach Eingang der Anmeldung vergeben. Weitere Informationen und Anmeldung unter www.aerzteball.ch oder info[at]aerzteball.ch 